\title{
Periodic phase separation: the periodic Cahn-Hilliard and isoperimetric problems
}

\author{
RUSTUM CHOKSI ${ }^{\dagger}$ \\ Department of Mathematics, Simon Fraser University, Burnaby, BC, Canada V5A 1S6 \\ AND \\ PETER STERnBerG \\ Department of Mathematics, Indiana University, Bloomington, IN 47405, USA
}

[Received 17 November 2005 and in revised form 3 April 2006]

\begin{abstract}
We consider two well known variational problems associated with the phenomenon of phase separation: the isoperimetric problem and minimization of the Cahn-Hilliard energy. The two problems are related through a classical result in $\Gamma$-convergence and we explore the behavior of global and local minimizers for these problems in the periodic setting. More precisely, we investigate these variational problems for competitors defined on the flat 2- or 3-torus. We view these two problems as prototypes for periodic phase separation. We give a complete analysis of stable critical points of the 2-d periodic isoperimetric problem and also obtain stable solutions to the 2-d and 3-d periodic Cahn-Hilliard problem. We also discuss some intriguing open questions regarding triply periodic constant mean curvature surfaces in 3-d and possible counterparts in the Cahn-Hilliard setting.
\end{abstract}

\section{Introduction}

Many physical systems exhibit a phase separation that, according to experiments, can be described roughly as follows:

- The phase separation is periodic on some fixed scale (often a mesoscopic scale much less than the sample size).

- Within a period cell, the structure appears to minimize surface area between the two phases.

Macroscopic phase separation (sometimes dubbed spinodal decomposition), i.e. phase separation on a scale comparable to the system size, has been the topic of extensive mathematical study (see for example [17, 26, 36], and the references therein). A simple model, in the spirit of van der Walls and Landau, was proposed in 1958 by Cahn and Hilliard [9]. The model is based upon minimizing the free energy

$$
\int_{\Omega}\left(\frac{\epsilon^{2}}{2}|\nabla u|^{2}+W(u)\right) \mathrm{d} x,
$$

where the order parameter $u$ represents the relative concentration of one of the two phases and obeys the mass conservation $\int_{\Omega} u \mathrm{~d} x$ fixed; $W$ is a nonnegative double-well preferring pure phases (e.g.

\footnotetext{
${ }^{\dagger}$ Research partially supported by NSERC Discovery Grants, Canada. E-mail: choksi@math.sfu.ca

${ }^{\ddagger}$ Research partially supported by NSF DMS-0401328 and the Institute for Mathematics and its Applications at the University of Minnesota. E-mail: sternber@indiana.edu
} 
$W( \pm 1)=0)$; and the small parameter $\epsilon$ represents the interfacial thickness at a transition between the phases. It is straightforward to see that the minimum energy of $[1.1)$ is $O(\epsilon)$ and hence it is natural to scale (1.1) by $1 / \epsilon$. With this rescaling, it has been shown rigorously (cf. [36, 53]) that minimizers of (1.1) converge to minimizers of a sharp-interface, pure perimeter problem. Because of the conservation of the total mass for the order parameter, the limit problem is an isoperimetric problem in which solutions possess phase boundaries of constant mean curvature (CMC). Hence, a natural approach to study periodic phase separation is via the periodic Cahn-Hilliard ( $\mathrm{PCH})$ and isoperimetric problems (PIP) whereby the physical domain is taken to be the $n$-dimensional flat torus.

One would expect these problems on a flat torus to be well understood. However, many questions remain open. Moreover, while the periodic isoperimetric problem has been the focus of much attention in the geometry community (see [29], [49] and the references therein), to our knowledge, the associated periodic Cahn-Hilliard problem, in particular the study of complex triply periodic local minimizers, has been ignored 1 This is particularly surprising given that, as we shall argue, the possible geometric structure of (local) minimizers in 3-d is far more complex and vast than with other well studied boundary conditions.

A physical paradigm for periodic phase separation is provided by microphase separation of diblock copolymers (cf. [5, 6, 56]). A diblock copolymer is a linear-chain molecule consisting of two sub-chains joined covalently to each other. One of the sub-chains is made of monomers of type $\mathrm{A}$ and the other of type B. Below a critical temperature, even a weak repulsion between unlike monomers $\mathrm{A}$ and $\mathrm{B}$ induces a strong repulsion between the sub-chains, causing the sub-chains to segregate. A macroscopic segregation, whereby the sub-chains detach from one another, cannot occur because the chains are chemically bonded: rather, a phase separation on a mesoscopic scale with A and B-rich domains emerges. The observed mesoscopic domains, illustrated in Figure 1, are highly regular periodic structures; for example lamellas, spheres, cylindrical tubes, and single and double gyroids (see for example, [6] and the references therein). These, and many of the other observed structures, strongly resemble triply periodic constant mean curvature (CMC) surfaces. This fact is well documented in both the science and mathematics literature (cf. [5, 6, 30, 49, 56]). Hence, focusing on one periodic cell, one may naturally regard (PCH) and (PIP) as toy problems for microphase separation of diblock copolymers.

On the other hand, it would seem that any model for these polymer configurations cannot simply be based solely on minimization of A-B monomer interfaces. Indeed, a density functional theory, first proposed by Ohta and Kawasaki [42], entails the minimization of a nonlocal CahnHilliard like energy (cf. [11, 40]) whereby the standard Cahn-Hilliard free energy is augmented by a long-range interaction term-associated with the connectivity of the sub-chains in the diblock copolymer macromolecule. This term is proportional to an interaction material parameter related to the length of the copolymer chain. We refer to this nonlocal counterpart as the nonlocal CahnHilliard problem (see formula 6.37) ) and its sharp interface version as the nonlocal isoperimetric problem (see (6.38). We will discuss them briefly in Section 6, but a subsequent paper [12] is devoted entirely to their study. We stress two important facts concerning these nonlocal functionals. When the interaction parameter is sufficiently large, energetic competitions between the perimeter and nonlocal terms sets a new mesoscopic length scale (smaller than the physical domain size) for minimizers. Moreover, heuristic, numerical, and analytical results suggest that minimizers are

\footnotetext{
1 In [18], the authors pursue an interesting analysis of the two-dimensional periodic Cahn-Hilliard problem, but from the standpoint of bifurcation theory in a much different vein than is taken here.
} 


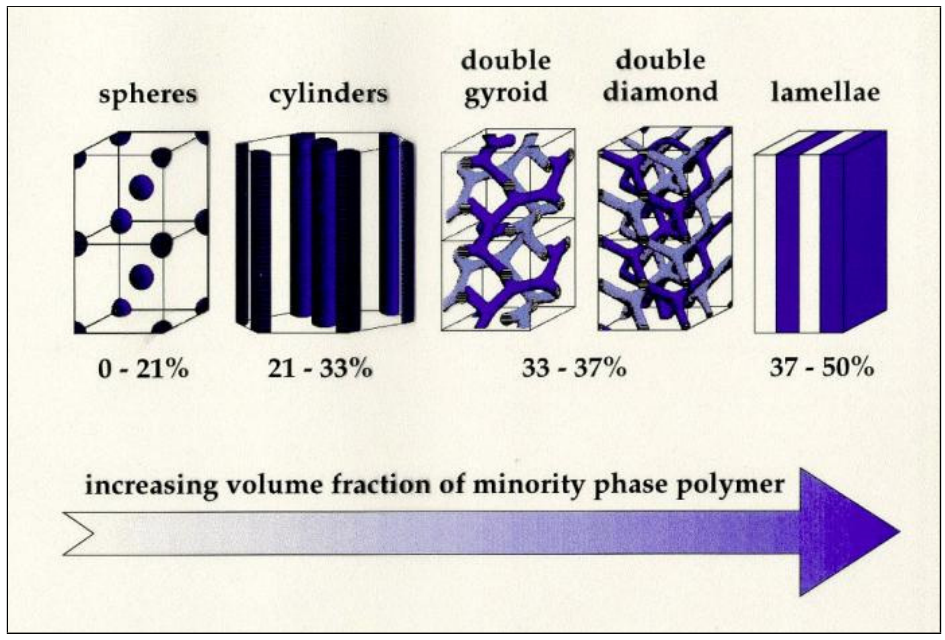

FIG. 1. Periodic phase separation. Cartoon from Edwin Thomas' talk at MSRI 1999-taken from reference [49].

nearly periodic (on this smaller length scale), regardless of the boundary conditions adopted on the physical domain (cf. [2, 3, 10, 39, 42, 44, 55, 60]). As for the geometry of minimizers, it is well known (cf. [34, 52]) that vanishing first variation of the area functional with respect to the volume constraint reduces to constant mean curvature for the phase boundary. On the other hand, with the nonlocal term, vanishing first variation does not imply CMC (see [12] for a full treatment of the first and second variations) and so not surprisingly, there are local minimizers which do not have CMC (cf. [39, 45]). Nonetheless, accepting both the validity of nonlocal model and the many experimental observations, it would seem that the effect of the nonlocal interactions within a period cell is minimal; rather, surface tension is the driving force (numerical evidence supports this claim-cf. [55]). Hence, in addition to being of interest in their own right, the local periodic problems focused on in the present article seem quite pertinent to the diblock problem as well.

We have in mind two primary goals in our investigation of the periodic Cahn-Hilliard and isoperimetric problems:

(i) Address the issue of global minimizers in two and three space dimensions. We will document what is known for the periodic isoperimetric problem, and with the tool of $\Gamma$-convergence (cf. [7]), we will exploit the work of geometers to easily obtain analogous stable solutions to the periodic Cahn-Hilliard problem. While these results are not difficult to prove, as far as we are aware, they have not been previously observed in the vast literature on the subject.

(ii) A more novel goal pertains to local minimizers. For the periodic isoperimetric problem, we address the possibility that strict stability (e.g. an appropriate positive lower bound on the second variation) implies an appropriate notion of strict local minimality with respect to small $L^{1}$ perturbations. A similar conjecture in the stronger $C^{1}$ topology and with Dirichlet boundary conditions is addressed in [18]. As we describe in Section 5, there is a wealth of complex triply periodic stable surfaces. Many physics papers (see for example [1, 4, 5, 56, 13, 15) allude to these surfaces and many papers in geometry (see for example [33, 50]) are devoted to their study. The natural question arises as to whether their structure is seen at the diffuse 
interface (Cahn-Hilliard) level. One route to a positive answer rests on proving this connection between strict stability and strict local minimality in three dimensions. Here we address the conjecture in two dimensions. We exhaust the list of all possible critical points to see which are unstable, which are stable and which are $L^{1}$-local minimizers. We work in such a weak topology due to our wish to invoke $\Gamma$-convergence results in drawing conclusions about the Cahn-Hilliard setting. Of course, in two dimensions, criticality alone leads immediately to curves with constant curvature (i.e. circles and lines), thus severely limiting the number of possible candidates. In the future, we hope to address whether in 3-d, some form of strict stability implies strict $L^{1}$-local minimality. While this will naturally require an entirely new machinery, our work here in 2-d lends support to the conjecture that the answer is indeed yes.

From a geometer's point of view, one might argue as to the relevance and virtue of the diffuse interface version. To this viewpoint, however, we respond that numerics are not easily performed with sharp interface energetic formulations, while a standard gradient flow approach to the diffuse interface energy gives a parabolic PDE (the well known Cahn-Hilliard equation), for which numerical algorithms are fairly well developed. In the same vein, one can view the periodic Cahn-Hilliard problem as a kind of regularization of the sharp interface problem, with the hope that the diffuse problem might shed light on some of the many remaining open questions regarding the 3-d periodic isoperimetric problem. In any event, we hope that this article will stimulate interest in both these important geometric and analytical problems, and, in particular, their interaction. The paper is organized as follows. In Section 2, we review some basic concepts from geometric measure theory. In Section 3 we formally introduce the problems, and note their connection via the theory of $\Gamma$-convergence. Global minimizers are addressed in Section 4 and local minimizers in Section 5. In Section 6, we briefly address the nonlocal analogues but only in the case where the nonlocalities are small. We conclude in Section 7 with some remarks.

\section{Some preliminaries}

We will require a few preliminaries from geometric measure theory. For further background, we refer the reader to [16, 20, 52].

For $n \geqslant 1$ (usually $n=2,3$ ), let $\mathbb{T}^{n}(n=2$ or 3 ) denote the $n$-dimensional flat torus. In particular we identify two points $\left(x_{1}, \ldots, x_{n}\right)=\left(y_{1}, \ldots, y_{n}\right)$ iff $x_{i}=y_{i}+k_{i}$ for some integers $k_{i}$.

Let $H^{1}\left(\mathbb{T}^{n}\right)$ denote the Sobolev space obtained by taking the closure under the Sobolev norm on $(-1 / 2,1 / 2)^{n}$ of periodic $C^{\infty}\left(\mathbb{R}^{n}\right)$ functions, i.e. functions $\varphi$ which satisfy

$$
\varphi\left(x_{1}+k_{1}, \ldots, x_{n}+k_{n}\right)=\varphi\left(x_{1}, \ldots, x_{n}\right) \quad \text { for all integers } k_{i}, i=1, \ldots n .
$$

Let $B V\left(\mathbb{T}^{n}\right)$ denote the set of all $L^{1}\left(\mathbb{T}^{n}\right)$ functions $u$ such that the total variation

$$
\int_{\mathbb{T}^{n}}|\nabla u|:=\sup _{\sigma} \int_{\mathbb{T}^{n}} u \operatorname{div} \sigma \mathrm{d} x<\infty,
$$

where the supremum is taken over all vector fields $\sigma \in C^{1}\left(\mathbb{R}^{n}, \mathbb{R}^{n}\right)$ whose components each satisfy (2.2), and such that $|\sigma| \leqslant 1$.

If $S \subset \mathbb{T}^{n}$, one says $S$ is of finite perimeter in $\mathbb{T}^{n}$ if the characteristic function of $S, \chi_{S}$, lies in $B V\left(\mathbb{T}^{n}\right)$. Then one defines the perimeter of $S$ as

$$
P\left(S, \mathbb{T}^{n}\right):=\int_{\mathbb{T}^{n}}\left|\nabla \chi_{S}\right|
$$


When we wish to be specific about functions on the torus, it is often convenient to give coordinates to $\mathbb{T}^{n}$ via the cube

$$
Q_{n}:=[-1 / 2,1 / 2)^{n} \subset \mathbb{R}^{n},
$$

but note that the spaces $H^{1}\left(\mathbb{T}^{n}\right)$ and $B V\left(\mathbb{T}^{n}\right)$ are not simply $H^{1}\left(Q_{n}\right)$ and $B V\left(Q_{n}\right)$ respectively.

A set $E \subset \mathbb{T}^{n}$ of finite perimeter is of course only defined up to a set of Lebesgue measure zero. In order to identify a useful representative for such an equivalence class, we introduce the density of $E$ at a point $x$ given by the limit

$$
D(E, x)=\lim _{r \rightarrow 0} \frac{|E \cap B(x, r)|}{|B(x, r)|} .
$$

Here $B(x, r)$ denotes the $n$-dimensional ball centered at $x$ of radius $r$. One defines the measuretheoretic interior of a set $E$ of finite perimeter as the set of all those $x$ for which $D(E, x)=1$. Similarly, the measure-theoretic exterior is taken to be the set of all those $x$ for which $D(E, x)=0$. Then the measure-theoretic boundary of $E$, denoted by $\partial_{M} E$, consists of those $x$ for which either $0<D(E, x)<1$ or the limit in 2.5 fails to exist. Clearly, $\partial_{M} E \subset \partial E$, where $\partial E$ denotes the topological boundary. When necessary, in this paper we will choose the measure-theoretic interior of $E$ as its representative.

Denoting $(n-1)$-dimensional Hausdorff measure by $\mathcal{H}^{n-1}$, it is well known that

$$
E \text { is of finite perimeter if and only if } \mathcal{H}^{n-1}\left(\partial_{M} E\right)<\infty
$$

and that

$$
P\left(E, \mathbb{T}^{n}\right)=\mathcal{H}^{n-1}\left(\partial_{M} E\right)=\mathcal{H}^{n-1}\left(\partial^{*} E\right),
$$

where $\partial^{*} E$ is the reduced boundary of $E$, consisting of all those boundary points of $E$ possessing a measure-theoretic normal (cf. [16, Section 4.5]). Furthermore, the rectifiability of $\partial_{M} E$ means that one has the useful decomposition

$$
\partial_{M} E=\bigcup_{j=0}^{\infty} \Gamma_{j}
$$

where $\mathcal{H}^{n-1}\left(\Gamma_{0}\right)=0$ and $\Gamma_{j}$ is the image of a Lipschitz map for $j \geqslant 1$. As the $\Gamma_{j}$ are boundary components, it can then be shown for the case $n=2$ that, in particular, the $\Gamma_{j}$ are given by closed Lipschitz curves for $j \geqslant 1$ ([16, 4.2.25]).

At times, it will also be useful to us to view a set of finite perimeter $E \subset \mathbb{R}^{2}$ as a 2-current of multiplicity one. Recall that a 2-current is simply a bounded linear functional acting on 2-forms. The mass of a $k$-current defined on a set $\Omega$ is given by

$$
M(T) \equiv \sup _{\left\{\varphi \in \mathcal{D}^{k}(\Omega):|\varphi| \leqslant 1\right\}}|T(\varphi)|,
$$

where $\mathcal{D}^{k}(\Omega)$ denotes the set of smooth, compactly supported $k$-forms on $\Omega$. The boundary of an $k$-current $T$, denoted by $\partial T$, is the $(k-1)$-current defined by the relation

$$
\partial T(\varphi)=T(d \varphi) \quad \text { for all } \varphi \in \mathcal{D}^{k-1}(\Omega),
$$

where $d \varphi$ represents the $k$-form obtained by exterior differentiation of $\varphi$. 
For a 1-current $T$, we also wish to recall the notion of the slice of a current by a Lipschitz function $f: \Omega \rightarrow \mathbb{R}^{1}$ (cf. [52, p. 155]). If $T$ is described by a 1-rectifiable set $\Gamma$, a tangent vector $\xi: \Gamma \rightarrow \mathbb{R}^{2}$ and a multiplicity $m: \Gamma \rightarrow \mathbb{Z}$, then the slice of $T$ by $f$ at a value $t \in \mathbb{R}^{1}$, denoted by $\langle T, f, t\rangle$, is a 0 -current defined for $H^{1}$-a.e. $t \in \mathbb{R}^{1}$. Letting $\nabla^{\Gamma} f$ denote the gradient of $f$ relative to $\Gamma$ and $\Gamma_{+} \equiv\left\{x \in \Gamma:\left|\nabla^{\Gamma} f(x)\right|>0\right\}$, the slice is supported on the countable set of points $f^{-1}(t) \cap \Gamma_{+}$, carries orientation \pm 1 according to the relation $\xi(x)= \pm \nabla^{\Gamma} f(x) /\left|\nabla^{\Gamma} f(x)\right|$, and carries multiplicity $m\left\lfloor\Gamma_{+}\right.$. Of most significance to our purposes is the inequality

$$
\int_{-\infty}^{\infty} M(\langle T, f, t\rangle) d t \leqslant \sup _{x \in \Gamma}\left|\nabla^{\Gamma} f(x)\right| M(T) .
$$

\section{The connection between (PCH) and (PIP) via $\Gamma$-convergence}

We first formulate the two problems. For $u \in L^{1}\left(\mathbb{T}^{n}\right), m \in(-1,1)$, and $\epsilon>0$, we define the Cahn-Hilliard energy as

$$
E_{\epsilon}(u):= \begin{cases}\int_{\mathbb{T}^{n}}\left(\frac{1}{4 \epsilon}\left(u^{2}-1\right)^{2}+\frac{\epsilon}{2}|\nabla u|^{2}\right) \mathrm{d} x & \text { if } u \in H^{1}\left(\mathbb{T}^{n}\right), \int_{\mathbb{T}^{n}} u=m, \\ +\infty & \text { otherwise. }\end{cases}
$$

By the Periodic Cahn-Hilliard Problem we mean

(PCH) Minimize $E_{\epsilon}(u)$ over all $u \in L^{1}\left(\mathbb{T}^{n}\right)$.

We also define, for $c_{0}=2 \sqrt{2} / 3$, a limit energy of the form

$$
E_{0}(u):= \begin{cases}c_{0} P\left(\{x: u(x)=1\}, \mathbb{T}^{n}\right) & \text { if } u \in B V\left(\mathbb{T}^{n}\right),|u|=1 \text { a.e., } \int_{\mathbb{T}^{n}} u=m, \\ +\infty & \text { otherwise, }\end{cases}
$$

and introduce the associated sharp interface minimization problem:

$(\mathrm{PIP})^{\prime} \quad$ Minimize $E_{0}(u)$ over all $u \in L^{1}\left(\mathbb{T}^{n}\right)$.

It is sometimes more convenient to rephrase the limit problem in terms of sets of finite perimeter. To a given $u_{0} \in B V\left(\mathbb{T}^{n},\{ \pm 1\}\right)$ with $\int_{\mathbb{T}^{n}} u_{0}=m$, one naturally associates a set of finite perimeter

$$
A:=\left\{x \in \mathbb{T}^{n}: u_{0}(x)=1\right\}, \quad|A|=a:=\frac{m+1}{2} .
$$

Conversely, to a given set of finite perimeter $A$ with $|A|=a$, one naturally associates $u_{0} \in$ $B V\left(\mathbb{T}^{n},\{ \pm 1\}\right)$ given by

$$
u_{0}(x)= \begin{cases}1 & \text { if } x \in A \\ -1 & \text { if } x \in A^{c}\end{cases}
$$

Clearly $\int_{\mathbb{T}^{n}} u_{0}=m=2 a-1$. Thus (PIP)' is equivalent to what is known as the Periodic Isoperimetric Problem:

(PIP) Minimize $P\left(A, \mathbb{T}^{n}\right)$ over $A \subset \mathbb{T}^{n}$ with finite perimeter $|A|=a$. 
We note that through the (weak) compactness and lower semicontinuity properties of $H^{1}\left(\mathbb{T}^{n}\right)$ and $B V\left(\mathbb{T}^{n}\right)$, global minimizers of both (PCH) and (PIP) are easily obtained via the direct method in the calculus of variations. Proposition 3.1 supplies the well known connection between the two problems via $\Gamma$-convergence.

Proposition 3.1 The sequence $E_{\epsilon} \Gamma$-converges to $E_{0}$ in $L^{1}\left(\mathbb{T}^{n}\right)$ (cf. [7]). That is,

for every $v \in L^{1}\left(\mathbb{T}^{n}\right)$ and every sequence $\left\{v_{\varepsilon}\right\}$ converging to $v$ in $L^{1}$

one has $\liminf _{\varepsilon \rightarrow 0} E_{\varepsilon}\left(v_{\varepsilon}\right) \geqslant E_{0}(v)$

and

for every $w \in L^{1}\left(\mathbb{T}^{n}\right)$ there exists a sequence $\left\{w_{\varepsilon}\right\} \subset L^{1}\left(\mathbb{T}^{n}\right)$ such that $w_{\varepsilon} \rightarrow w$ in $L^{1}$ and $\lim _{\varepsilon \rightarrow 0} E_{\varepsilon}\left(w_{\varepsilon}\right)=E_{0}(w)$.

Consequently, for any sequence $\left\{u_{\epsilon_{j}}\right\}$ of minimizers of $(\mathrm{PCH})$, there exists a subsequence (not relabeled) such that

$$
u_{\epsilon_{j}} \rightarrow u_{0} \quad \text { in } L^{1}\left(\mathbb{T}^{n}\right),
$$

where $u_{0}$ is a minimizer of (PIP)'.

Proof. The fact that $E_{\epsilon} \Gamma$-converges to $E_{0}$ follows directly from the Modica-Mortola theorem ([36, 37]). Moreover, the usual compactness argument also applies directly to produce a subsequence $u_{\epsilon_{j}}$ converging in $L^{1}\left(\mathbb{T}^{n}\right)$ to a limit $u_{0} \in B V\left(\mathbb{T}^{n}\right)$ with $\left|u_{0}\right|=1$ a.e. and $\int_{\mathbb{T}^{n}} u_{0} \mathrm{~d} x=m$. Finally, for any $w \in L^{1}\left(\mathbb{T}^{n}\right)$ condition 3.14 guarantees the existence of a sequence $w_{\epsilon_{j}}$ converging to $w$ in $L^{1}\left(\mathbb{T}^{n}\right)$ such that

$$
\lim E_{\epsilon_{j}}\left(w_{\epsilon_{j}}\right)=E_{0}(w)
$$

Hence by (3.13) we have

$$
E_{0}\left(u_{0}\right) \leqslant \liminf E_{\epsilon_{j}}\left(u_{\epsilon_{j}}\right) \leqslant \liminf E_{\epsilon_{j}}\left(w_{\epsilon_{j}}\right)=E_{0}(w),
$$

so $u_{0}$ is a minimizer of $E_{0}$.

We also wish to explore the possibility of local minimizers to (PCH) and (PIP) and to investigate their relationship to each other via $\Gamma$-convergence. By an $L^{1}$-local minimizer of $(\mathrm{PCH})$ we mean an admissible (finite energy) function $u_{\epsilon}$ with the property that there exists a $\delta>0$ such that

$$
E_{\epsilon}\left(u_{\epsilon}\right) \leqslant E_{\epsilon}(v) \quad \text { for all admissible } v \text { with } 0<\left\|u_{\epsilon}-v\right\|_{L^{1}\left(\mathbb{T}^{n}\right)}<\delta .
$$

Of course, such a function $u_{\varepsilon}$ will automatically locally minimize in the stronger $H^{1}$-topology and will in particular satisfy the Euler-Lagrange equation associated with criticality.

By an $L^{1}$-local minimizer of (PIP)' we mean an admissible (finite energy) function $u_{0}$ with the property that there exists a $\delta>0$ such that

$$
E_{0}\left(u_{0}\right) \leqslant E_{0}(v) \quad \text { for all admissible } v \text { with } 0<\left\|u_{0}-v\right\|_{L^{1}\left(\mathbb{T}^{n}\right)}<\delta .
$$

In either case, if the inequality for the energies is strict, we use the term isolated $L^{1}$-local minimizer. The main result of [35] asserts that near isolated $L^{1}$-local minimizers of $E_{0}$ one can find local minimizers of $E_{\varepsilon}$ (the different boundary conditions are irrelevant here). Unfortunately, in light 
of the translation invariance of the periodic isoperimetric problem, the local minimizers we will consider are never isolated. Hence, we must invoke this slight generalization of the result from [35]. In what follows, we use $d_{L^{1}}(v, S)$ to denote the $L^{1}$ distance between a function $v \in L^{1}\left(\mathbb{T}^{n}\right)$ and a set $S \subset L^{1}\left(\mathbb{T}^{n}\right)$, i.e.

$$
d_{L^{1}}(v, S):=\inf _{u \in S}\|v-u\|_{L^{1}\left(\mathbb{T}^{n}\right)} .
$$

Proposition 3.2 Suppose $S \subset L^{1}\left(\mathbb{T}^{n}\right)$ is a set of locally minimizing critical points of $E_{0}$ in the sense that there exist positive numbers $M$ and $\delta$ such that for all $u \in S$ one has

$$
E_{0}(v)>E_{0}(u)=M \quad \text { whenever } \quad 0<d_{L^{1}}(v, S)<\delta .
$$

Suppose furthermore that $S$ is compact in $L^{1}\left(\mathbb{T}^{n}\right)$; that is, for every sequence $\left\{u_{j}\right\} \subset S$ suppose there exists a subseqence $\left\{u_{j_{k}}\right\}$ converging in $L^{1}$ to a limit $u \in S$. Then there exists a value $\varepsilon_{0}>0$ and, for all $\varepsilon<\varepsilon_{0}$, a family of $L^{1}$-local minimizers $\left\{u_{\varepsilon}\right\}$ of $E_{\varepsilon}$ such that $d\left(u_{\varepsilon}, S\right) \rightarrow 0$ as $\varepsilon \rightarrow 0$. Furthermore, for any sequence $\varepsilon_{j} \rightarrow 0$, there exists a subsequence $\left\{\varepsilon_{j_{k}}\right\}$ and an element $u$ of $S$ such that $u_{\varepsilon_{j_{k}}} \rightarrow u$ in $L^{1}$.

If the hypothesis of Proposition 3.2 holds, we call $v$ a strict $L^{1}$-local minimizer.

Proof. This modification of the result from [35] is fairly well known but for the sake of selfcontainment, we sketch its proof here. We begin by fixing any positive number $\delta_{1}<\delta$ and for each $\varepsilon>0$ we define $u_{\varepsilon}$ as any solution to the minimization problem

$$
\inf _{\left\{v: d(v, S) \leqslant \delta_{1}\right\}} E_{\varepsilon}(v) .
$$

The existence of such a minimizer follows via the direct method by utilizing standard compactness and lower semicontinuity properties. Suppose, by way of contradiction, that there exists a subsequence $\left\{u_{\varepsilon_{j}}\right\}$ and a positive number $\gamma \leqslant \delta_{1}$ such that

$$
d\left(u_{\varepsilon_{j}}, S\right) \geqslant \gamma
$$

Invoking the usual compactness property for sequences of functions of uniformly bounded CahnHilliard energy, we can pass to an $L^{1}$-convergent subsequence (still denoted by $u_{\varepsilon_{j}}$ ):

$$
u_{\varepsilon_{j}} \rightarrow v \text { for some } v \in L^{1} .
$$

Furthermore, the condition $\gamma \leqslant d\left(u_{\varepsilon_{j}}, S\right) \leqslant \delta_{1}$ and the compactness of $S$ imply that there exists a function $w \in S$ with $0<\gamma \leqslant\|v-w\|_{L^{1}} \leqslant \delta_{1}$. Then, invoking 3.13) and 3.14), we have

$$
E_{0}(v) \leqslant \liminf E_{\varepsilon_{j}}\left(u_{\varepsilon_{j}}\right) \leqslant \lim E_{\varepsilon_{j}}\left(w_{\varepsilon_{j}}\right)=E_{0}(w)
$$

contradicting 3.17).

The final claim of this proposition follows readily from the compactness of $S$.

We conclude this section with a remark about regularity for local minimizers of (PIP)'. It is well known that any minimizer of (PIP), in fact any $L^{1}$-local minimizer, must have a regular boundary. This is stated more precisely in the following result of Gonzalez, Massari, and Tamanini [21]. The presence of periodic boundary conditions here makes no difference in their proof. 
THEOREM 3.3 Let $n<8$. If $u_{0}$ is an $L^{1}$-local minimizer of (PIP)' with associated set $A$ of finite perimeter, then $\partial A$ is an analytic $(n-1)$-dimensional manifold.

A similar result holds in higher space dimension except for a singular set with Hausdorff dimension at most $n-8$ (cf. [21]). With the regularity in hand, a classical calculation (see for example [34, 52]) implies that the phase boundary associated with any $L^{1}$-local minimizer of (PIP)' has constant mean curvature-a direct consequence of vanishing first variation.

\section{Global minimizers of (PCH) and (PIP)}

\subsection{Global minimizers in 2-d}

The following theorem is surely classical. We present the proof given by Howards, Hutchings and Morgan in [31].

THEOREM 4.1 Let $n=2$. The minimizers of (PIP) are either a disc or a strip (i.e. the region enclosed by two parallel 1-tori). In fact, the minimizer is

- a disc if either $0<a \leqslant 1 / \pi$ or $1-1 / \pi \leqslant a \leqslant 1$;

- a strip (two parallel $1-\mathrm{d}$ tori) if $1 / \pi \leqslant a \leqslant 1-1 / \pi$.

Proof. That a minimizer must exist follows by the direct method of the calculus of variations, in particular the weak compactness property of the space $B V$ and the weak lower semicontinuity of the total variation. Consider such a minimizer. By Theorem 3.3 , its boundary must be regular, and hence must be of constant mean curvature. In two dimensions this forces the boundary to consist of either a (finite) union of circles or of line segments. In the case of circles, there can only be one; otherwise one could translate one circle towards another, without changing the perimeter or the enclosed area, until the two circles touch. This would produce a singular minimizer which violates the regularity. For the case of line segments, it is clear that the optimal situation occurs when the boundary consists of exactly two parallel line segments. The regimes for the two cases are obtained by considering the two critical values of $a$ : when $2 \pi \sqrt{a / \pi}=2$ and when $2 \pi \sqrt{(1-a) / \pi}=2$.

We can now argue that for $\varepsilon$ small, the minimizers of (PCH) must exhibit a profile asymptotic to the solutions of (PIP). To this end, we introduce functions $u_{L}: \mathbb{T}^{2} \rightarrow \mathbb{R}^{1}, u_{D}^{+}: \mathbb{T}^{2} \rightarrow \mathbb{R}^{1}$ and $u_{D}^{-}: \mathbb{T}^{2} \rightarrow \mathbb{R}^{1}$ taking values \pm 1 on the set and its complement solving (PIP). (The subscript $L$ stands for lamellas and $D$ stands for disc.) Specifically, we define

$$
\begin{aligned}
& u_{L}(x, y):= \begin{cases}+1 & \text { if } 0<x<(m+1) / 4 \text { or }(3-m) / 4<x<1, \\
-1 & \text { if }(m+1) / 4<x<(3-m) / 4,\end{cases} \\
& u_{D}^{+}(x, y):= \begin{cases}+1 & \text { if }|(x, y)|>\sqrt{(1-m) / 2 \pi} \\
-1 & \text { if }|(x, y)|<\sqrt{(1-m) / 2 \pi}\end{cases} \\
& u_{D}^{-}(x, y):= \begin{cases}+1 & \text { if }|(x, y)|<\sqrt{(m+1) / 2 \pi} \\
-1 & \text { if }|(x, y)|>\sqrt{(m+1) / 2 \pi}\end{cases}
\end{aligned}
$$

Then we can establish 
COROLlary 4.2 Let $\left\{u_{\epsilon}\right\}$ denote any sequence of minimizers of (PCH).

- If $2 / \pi-1<m<1-2 / \pi$, then there exists a sequence $\left\{c_{\varepsilon}\right\}$ of numbers between $-1 / 2$ and $1 / 2$ and a sequence $\left\{k_{\varepsilon}\right\}$ with $k_{\varepsilon} \in\{0,1\}$ such that

$$
\tilde{u}_{\varepsilon} \rightarrow u_{L} \quad \text { in } L^{1}\left(\mathbb{T}^{2}\right)
$$

where $\tilde{u}_{\varepsilon}(x, y):=u_{\varepsilon}\left(R^{k_{\varepsilon}}\left((x, y)+c_{\varepsilon}(1,0)\right)\right)$ and $R$ represents a clockwise rotation by $\pi / 2$.

- If $-1<m<2 / \pi-1$, then there exists a sequence $\left\{\left(x_{\varepsilon}, y_{\varepsilon}\right)\right\} \subset \mathbb{T}^{2}$ such that

$$
\tilde{u}_{\varepsilon} \rightarrow u_{D}^{-} \quad \text { in } L^{1}\left(\mathbb{T}^{2}\right)
$$

where $\tilde{u}_{\varepsilon}(x, y):=\tilde{u}_{\varepsilon}\left(x-x_{\varepsilon}, y-y_{\varepsilon}\right)$.

- If $1-2 / \pi<m<1$, then there exists a sequence $\left\{\left(x_{\varepsilon}^{\prime}, y_{\varepsilon}^{\prime}\right)\right\} \subset \mathbb{T}^{2}$ such that

$$
\tilde{u}_{\varepsilon} \rightarrow u_{D}^{+} \quad \text { in } L^{1}\left(\mathbb{T}^{2}\right)
$$

where $\tilde{u}_{\varepsilon}(x, y):=\tilde{u}_{\varepsilon}\left(x-x_{\varepsilon}^{\prime}, y-y_{\varepsilon}^{\prime}\right)$.

Proof. The three cases are all handled in a similar manner. We will present the second case. The argument proceeds by contradiction, so we suppose there exists a number $\delta>0$ and a sequence $\left\{\varepsilon_{j}\right\} \rightarrow 0$ such that

$$
\inf _{\left(x^{\prime}, y^{\prime}\right) \in \mathbb{T}^{2}}\left\|u_{\varepsilon_{j}}\left(\cdot-\left(x^{\prime}, y^{\prime}\right)\right)-u_{D}^{-}\right\|_{L^{1}\left(\mathbb{T}^{2}\right)} \geqslant \delta .
$$

In light of the compactness result of Proposition 3.1 there is a further subsequence (which we still denote by $\left.\left\{\varepsilon_{j}\right\}\right)$ and an $L^{1}$-function $v$ minimizing $E_{0}$ such that $u_{\varepsilon_{j}} \rightarrow v$ in $L^{1}\left(\mathbb{T}^{2}\right)$. For $m \in$ $(-1,2 / \pi-1)$, Theorem 4.1 says that $v$ must take the form

$$
v(x, y):= \begin{cases}+1 & \text { if }\left|(x, y)-\left(x_{0}, y_{0}\right)\right|<\sqrt{(m+1) / 2 \pi}, \\ -1 & \text { if }\left|(x, y)-\left(x_{0}, y_{0}\right)\right|>\sqrt{(m+1) / 2 \pi},\end{cases}
$$

for some $\left(x_{0}, y_{0}\right) \in \mathbb{T}^{2}$. But then since $v(x, y)=u_{D}^{-}\left(x-x_{0}, y-y_{0}\right)$, we have

$$
\left\|u_{\varepsilon_{j}}\left(\cdot+\left(x_{0}, y_{0}\right)\right)-u_{D}^{-}\right\|_{L^{1}\left(\mathbb{T}^{2}\right)} \rightarrow 0,
$$

contradicting 4.21.

\subsection{Global minimizers in 3-d}

Surprisingly enough, there are few rigorous results yielding the solution to the three-dimensional periodic isoperimetric problem. Indeed, we know of only the following two results.

THEOREM 4.3 (Hadwiger [27]) If $a=1 / 2$ then any minimizer of (PIP) must be either a horizontal or vertical strip, i.e. two parallel 2-tori.

Theorem 4.4 (see, for example, Theorem 18 and the comments which follow in Ros [49]) For the value $a$ sufficiently small, any minimizer of (PIP) must be a sphere (ball).

A full result characterizing all minimizers of (PIP) for different $a$ remains an open problem in classical geometry. For our cubic flat torus $\mathbb{T}^{3}$, the following conjecture is well accepted and well tested (cf. [29, 49]): 
CONJECTURE 4.5 If $n=3$, minimizers of (PIP) are-in terms of the phase boundaries-either a sphere, (quotient of) a cylinder, or two parallel flat tori (i.e. lamellas).

For more general flat 3-tori, the conjecture is less clear and experiments using Brakke's Surface Evolver [8] suggest that there may well exist nonstandard global minimizers (A. Ros, personal correspondence, and [29]). We should mention that Ritore has shown that for any rectangular torus in 3-d and for any fixed volume fraction, the global minimizer must be one of five candidates including the three candidates listed above and two other CMC surfaces, a Lawson surface and a Schwarz surface (see [49, Section 1.5]).

Combining Theorems 4.3 and 4.4 with Proposition 3.1 we have the following results for the 3-d (PCH). For $(x, y, z) \in Q_{3}$, let

$$
\begin{aligned}
u_{L_{3}}(x, y, z) & := \begin{cases}+1 & \text { if }-1 / 2<x<-1 / 4 \text { or } 1 / 4<x<1 / 2, \\
-1 & \text { if }-1 / 4<x<1 / 4,\end{cases} \\
u_{S}^{+}(x, y, z) & := \begin{cases}+1 & \text { if }|(x, y, z)| \geqslant(3 / \pi(1-m))^{1 / 3} / 2, \\
-1 & \text { if }|(x, y, z)|<(3 / \pi(1-m))^{1 / 3} / 2,\end{cases} \\
u_{S}^{-}(x, y, z): & = \begin{cases}-1 & \text { if }|(x, y, z)| \geqslant(3 / \pi(1+m))^{1 / 3} / 2, \\
+1 & \text { if }|(x, y, z)|<(3 / \pi(1+m))^{1 / 3} / 2 .\end{cases}
\end{aligned}
$$

COROLLARY 4.6 Let $\left\{u_{\epsilon}\right\}$ denote any sequence of minimizers of (PCH).

- If $m=0(a=1 / 2)$, then there exists a sequence $\left\{c_{\varepsilon}\right\}$ of numbers between $-1 / 2$ and $1 / 2$ and a sequence $\left\{R_{\varepsilon}\right\}$ of rotations such that

$$
\tilde{u}_{\varepsilon} \rightarrow u_{L_{3}} \quad \text { in } L^{1}\left(\mathbb{T}^{3}\right)
$$

where $\tilde{u}_{\varepsilon}(x, y, z):=u_{\varepsilon}\left(R_{\varepsilon}\left((x, y, z)+c_{\varepsilon}(1,0,0)\right)\right)$ and $R_{\varepsilon}$ is either the identity, a rotation of $Q_{3}$ by $\pi / 2$ with respect to the $x$-axis, or a rotation of $Q_{3}$ by $\pi / 2$ with respect to the $y$-axis.

- If $m$ is sufficiently close to 1 (hence $a$ is sufficiently close to 1 ) then there exists a sequence $\left\{\left(x_{\varepsilon}, y_{\varepsilon}, z_{\varepsilon}\right)\right\} \subset \mathbb{T}^{3}$ such that

$$
\tilde{u}_{\varepsilon} \rightarrow u_{S}^{+} \quad \text { in } L^{1}\left(\mathbb{T}^{3}\right)
$$

where $\tilde{u}_{\varepsilon}(x, y, z):=\tilde{u}_{\varepsilon}\left(x-x_{\varepsilon}, y-y_{\varepsilon}, z-z_{\varepsilon}\right)$.

- If $m$ is sufficiently close to -1 (hence $a$ is sufficiently close to 0 ), then there exists a sequence $\left\{\left(x_{\varepsilon}^{\prime}, y_{\varepsilon}^{\prime}, z_{\varepsilon}^{\prime}\right)\right\} \subset \mathbb{T}^{3}$ such that

$$
\tilde{u}_{\varepsilon} \rightarrow u_{S}^{-} \quad \text { in } L^{1}\left(\mathbb{T}^{3}\right)
$$

where $\tilde{u}_{\varepsilon}(x, y, z):=\tilde{u}_{\varepsilon}\left(x-x_{\varepsilon}^{\prime}, y-y_{\varepsilon}^{\prime}, z-z_{\varepsilon}^{\prime}\right)$.

Proof. The proof is analogous to the proof of Corollary 4.2

\section{Local minimizers of (PCH) and (PIP)}

We have already defined the notion of an $L^{1}$-local minimizer. A notion closely related to local minimality is stability, i.e. positivity of the second variation. Since for (PIP) this is usually phrased 
in terms of the phase boundary, we define the notion of a stable surface. Let $n=2$ or 3 . For any smooth $\left(C^{2}\right)(n-1)$-surface $\Sigma$ in $\mathbb{T}^{n}$, we let $B_{\Sigma}$ denote the second fundamental form so that

$$
\left\|B_{\Sigma}\right\|^{2}=\sum_{i=1}^{n-1} \kappa_{i}^{2}, \quad \text { where } \kappa_{1}, \ldots, \kappa_{n-1} \text { are the principal curvatures. }
$$

Given a set $A \subset \mathbb{T}^{n}$ with smooth boundary $\Sigma$, we say $\Sigma$ is a stable surface if it minimizes the area up to second order under the volume constraint, meaning that

(i) $\Sigma$ has constant mean curvature (CMC),

(ii) $Q(f, f) \geqslant 0$ for any function $f$ in the Sobolev space $H^{1}(\Sigma)$ satisfying $\int_{\Sigma} f \mathrm{~d} \mathcal{H}^{n-1}=0$. Here $Q$ denotes the quadratic form

$$
Q(f, f)=\int_{\Sigma}\left(\left|\nabla_{\Sigma} f\right|^{2}-\left\|B_{\Sigma}\right\|^{2} f^{2}\right) \mathrm{d} \mathcal{H}^{n-1},
$$

and $\nabla_{\Sigma} f$ denotes the gradient of $f$ relative to $\Sigma$.

Conditions (i) and (ii) above are direct consequences of vanishing first and nonnegative second variations respectively (cf. [34, 52]). In light of the regularity theory laid out in Theorem 3.3, it follows from a direct calculation of the second variation that local minimizers of (PIP) have stable boundaries.

The natural question arises as to whether positive second variation (stability) implies local minimality with respect to small perturbations in some topology (e.g. $C^{0}, C^{1}, C^{\infty}$, or $L^{1}$ ). This question has been well explored in the context of minimal surfaces (see for example [41]). In the present context of the volume constraint, it has been addressed by Grosse-Brauckmann [22] who, building on work of White [59], shows local minimality with respect to $C^{0}$-close perturbations. Here we are concerned with $L^{1}$-perturbations since it is through this topology that we can make a connection with the diffuse interface problem (cf. Prop. 3.2p.

In three dimensions, there exist a remarkable collection of nonstandard CMC surfaces which have been shown to be stable on various 3-tori. Such surfaces include Schwarz' P and D surfaces and Schoen's gyroid surface (cf. [23-25, 33, 49-51]). Though exhaustive work has yet to be done for stable CMC surfaces, it is known that such surfaces must have genus at most 4 (cf. [48, 49]). These surfaces and related questions on stability have also appeared in the physics literature (see for example [1, 4, 5, 13,-15] and the references therein). Determining whether or not there exist corresponding diffuse-interface local minimizers to the Cahn-Hilliard problem rests on showing, in dimension three, that stable surfaces (or more precisely strictly stable with an appropriate positive lower bound on the second variation) correspond to strict $L^{1}$-local minimizers of (PIP) in the sense of Proposition 3.2. We believe they do but are not yet able to prove this.

In two dimensions, the situation is considerably simplified by the fact that the CMC condition reduces the consideration of eligible phase boundaries to either circles or lines. In the following two propositions we argue that a single horizontal strip and a single disc always locally minimize (PIP) in a sense strong enough to allow application of Proposition 3.2.

Proposition 5.1 Let $A_{0} \subset \mathbb{T}^{2}$ denote the strip whose restriction to the unit cube $Q_{2}$ occupies the set $\{(x, y):-a / 2<y<a / 2\}$ for some $a \in(0,1)$. Then $A_{0}$ is a local minimizer of (PIP) in the sense that for some positive $\delta$ one has the condition

$$
P\left(A_{0}, \mathbb{T}^{2}\right) \leqslant P\left(A, \mathbb{T}^{2}\right)
$$


for all sets of finite perimeter $A \subset \mathbb{T}^{2}$ satisfying

$$
\left|A_{0} \triangle A\right|<\delta \quad \text { and } \quad|A|=\left|A_{0}\right|=a .
$$

Furthermore, equality holds in (5.23) only when $A$ differs from $A_{0}$ by a translation.

Equivalently, if $u_{0}: \mathbb{T}^{2} \rightarrow \mathbb{R}^{1}$ is given by

$$
u_{0}(x, y)= \begin{cases}1 & \text { if }(x, y) \in A_{0}, \\ -1 & \text { if }(x, y) \in A_{0}^{c}\end{cases}
$$

and $S_{1} \subset L^{1}\left(\mathbb{T}^{2}\right)$ is given by

$$
S_{1}=\left\{u_{0}(x, y-c): c \in[-1 / 2,1 / 2]\right\},
$$

then for all $u \in S_{1}$ one has

$$
E_{0}(v)>E_{0}(u)=2 \quad \text { provided } \quad \int_{\mathbb{T}^{2}} v=\int_{\mathbb{T}^{2}} u_{0} \quad \text { and } \quad 0<d_{L^{1}}\left(v, S_{1}\right)<\delta .
$$

Proof. Let $A$ be a set of finite perimeter satisfying (5.24) and let $\lambda:=\min \{a / 4,1 / 2(1-a / 2)\}$. We will first argue that in a neighborhood of the top of the strip $A_{0}$, the boundary of $A$ must project almost fully onto the boundary of $A_{0}$. To this end, we introduce the set $S \subset(-1 / 2,1 / 2)$ given by

$$
S:=\left\{x \in(-1 / 2,1 / 2):(x, y) \in \partial_{M} A \text { for some } y \in(a / 2-\lambda, a / 2+\lambda)\right\} .
$$

Now for every $x \notin S$ note that the vertical segment $\{x\} \times(a / 2-\lambda, a / 2+\lambda)$ either lies entirely within $A$ or entirely outside of $A$. In either case, half of the line segment lies within the symmetric difference $A_{0} \triangle A$. Consequently we have

$$
\frac{1}{2} \lambda \mathcal{H}^{1}((-1 / 2,1 / 2) \backslash S) \leqslant\left|A_{0} \triangle A\right|<\delta .
$$

At this point we choose $\delta=\lambda^{2} / 8$ to conclude that

$$
\mathcal{H}^{1}(S) \geqslant 1-2 \delta / \lambda=1-\lambda / 4 .
$$

From here, our strategy will be to show that in the set $\{y>0\}$, the set $A$ has at least perimeter 1 . Combining this with a similar argument in $\{y<0\}$ will lead to the desired conclusion that the strip has least perimeter among nearby competitors $A$.

To pursue this, we quantify the number of intersections between $\partial_{M} A$ and horizontal lines above $y=1 / 2-\lambda$ and below $y=a / 2-\lambda$. We introduce the set $S^{\prime} \in(0,1 / 2)$ given by

$$
S^{\prime}:=\left\{y \in(0, \lambda) \cup(1 / 2-\lambda, 1 / 2):(x, y) \in \partial_{M} A \text { for some } x \in(-1 / 2,1 / 2)\right\},
$$

and distinguish two cases: either

$$
\mathcal{H}^{1}\left(S^{\prime}\right) \geqslant \lambda / 2
$$

or

$$
\mathcal{H}^{1}\left(S^{\prime}\right)<\lambda / 2 .
$$

First suppose that 5.29$\}$ occurs. Then combining 5.28$)$ and 5.29$\}$ we see that $\mathcal{H}^{1}\left(\partial_{M} A \cap\{y>0\}\right)$ $>1+\lambda / 4>1$. Assuming (5.30) holds, consider the collection of all curves $\Gamma_{j}$ in the decomposition 
2.6) of $\partial_{M} A$ such that $(x, y) \in \Gamma_{j}$ for some $x \in S$. Using the definition of $S^{\prime}$ it must then be the case that any $\Gamma_{j}$ in this set is a closed curve on $\mathbb{T}^{2}$ that avoids the sets $\{y<\lambda / 2\}$ and $\{y>1 / 2-\lambda / 2\}$. If $\Gamma_{j}$ is homotopically trivial then its length must be at least double its projection onto $\{y=a / 2\}$. Furthermore, if all curves $\Gamma_{j}$ in this collection are homotopically trivial, then by this reasoning condition 5.28 would imply that the total length of these curves would exceed $2-\lambda / 2>1$. Finally, if at least one $\Gamma_{j}$ in this collection fails to be homotopically trivial, then it would have to span the unit square $Q_{2}$ from left to right, making its total length greater than 1 (unless it was parallel to $y=a / 2$, in which case its length would equal 1).

The upshot of pursuing these two cases is that necessarily the total perimeter of $A$ in the set $\{y>0\}$ exceeds 1 unless $\partial_{M} A$ consists of a horizontal segment, making its length exactly 1 . Since the same line of reasoning holds for $\partial_{M} A \cap\{y<0\}$, we conclude that $P\left(A, \mathbb{T}^{2}\right)>P\left(A_{0}, \mathbb{T}^{2}\right)=2$ unless $A$ is a translate of $A_{0}$.

COROLlary 5.2 There exists a value $\varepsilon_{0}>0$ and a sequence $\left\{u_{\varepsilon}\right\}$ of local minimizers of $E_{\varepsilon}$ such that $d_{L^{1}}\left(u_{\varepsilon}, S_{1}\right) \rightarrow 0$ as $\varepsilon \rightarrow 0$, where the set $S_{1}$ is given by 5.26 . Furthermore, for any sequence $\left\{\varepsilon_{j}\right\} \rightarrow 0$, there exists a subsequence $\left\{\varepsilon_{j_{k}}\right\}$ and an element $u$ of $S_{1}$ such that $u_{\varepsilon_{j_{k}}} \rightarrow u$ in $L^{1}$.

Proof. The corollary follows immediately from Propositions 3.2 and 5.1

REMARK 5.3 The proof of $L^{1}$-local minimality of a single horizontal, or for that matter, vertical strip given in Proposition 5.1 can be immediately adapted to the case where the set $A_{0}$ consists of a finite number of horizontal or vertical strips. This is due to the fact that the argument is entirely local. More precisely, one could simply pick any particular component of $A_{0}$ and then select the tolerance $\delta$ to be sufficiently small in terms of the distance from this one strip to the next nearest strip and argue along the lines just presented. However, we hasten to add that through such an argument we would only conclude that this union of strips $A_{0}$ satisfies the conditions $(5.23)-(5.24)$. For multicomponent sets of strips, it is no longer the case that the only nearby competitors with the same perimeter are translates of $A_{0}$ as it is now possible to shrink one strip while expanding another so as to preserve the enclosed area. This extra freedom precludes the formation of a compact set analogous to the set of translates $S_{1}$ given by $(5.26)$ and so one cannot apply Proposition 3.2 to obtain diffuse counterparts in the Cahn-Hilliard setting to arbitrary collections of horizontal or vertical strips. We presume that this is not simply an indication of the failure of the method but that indeed no such local minimizers exist to Cahn-Hilliard.

REMARK 5.4 Within the class of critical points for the 2-d periodic isoperimetric problem having zero curvature, the only other case to consider would be sets $A \subset \mathbb{T}^{2}$ having boundary consisting of a finite union of parallel but not necessarily vertical or horizontal line segments. This is because if the boundary segments are not all parallel, singularities must be present given the periodicity of the torus. Even for competitors with parallel boundary segments, the slopes would have to be rational for such a configuration to be smooth and have finitely many components. Since its boundary has curvature identically zero, it is again immediately seen to be stable (cf. (5.22)). One could then also argue as in Proposition 5.1 that such a collection of strips will be an $L^{1}$-local minimizer of (PIP). The extra step here not present in the case of horizontal or vertical strips, however, is the observation that the only $L^{1}$ close competitors will be collections of parallel strips with boundary slopes equal to that of the collection in question. Otherwise, inevitably such a competitor would possess a different number of components, making it far away in $L^{1}$. We also mention that for slanted strips with multiple components, we cannot apply Proposition 3.2 (see Remark 5.3 above).

Next we show the desired local minimality property holds for any disc on $\mathbb{T}^{2}$. 
Proposition 5.5 For any $R<1$, the disc $B(0, R)$ is a local minimizer of (PIP) in $\mathbb{T}^{2}$ in the sense that for some positive $\delta$ one has the condition

$$
P\left(B(0, R), \mathbb{T}^{2}\right) \leqslant P\left(A, \mathbb{T}^{2}\right)
$$

for all sets of finite perimeter $A \subset \mathbb{T}^{2}$ satisfying

$$
|B(0, R) \triangle A|<\delta \quad \text { and } \quad|A|=|B(0, R)|=\pi R^{2} .
$$

Furthermore, equality holds in 5.31) only when $A$ differs from $B(0, R)$ by a translation.

Equivalently, if $u_{0}: \mathbb{T}^{2} \rightarrow \mathbb{R}^{1}$ is given by

$$
u_{0}(x, y)= \begin{cases}1 & \text { if }(x, y) \in B(0, R) \\ -1 & \text { if }(x, y) \in B(0, R)^{c}\end{cases}
$$

and $S_{2} \subset L^{1}\left(\mathbb{T}^{2}\right)$ is given by

$$
S_{2}=\left\{u_{0}\left(x-c_{1}, y-c_{2}\right): c_{1}, c_{2} \in[-1 / 2,1 / 2]\right\},
$$

then for all $u \in S_{2}$ one has

$$
E_{0}(v)>E_{0}(u)=2 \text { provided } \int_{\mathbb{T}^{2}} v=\int_{\mathbb{T}^{2}} u_{0} \quad \text { and } \quad 0<d_{L^{1}}\left(v, S_{2}\right)<\delta .
$$

Proof. Let $A$ be a set of finite perimeter satisfying (5.32). Our first goal is to argue that necessarily, $A$ has perimeter nearly $2 \pi R$ in the thin annulus $\mathcal{A}_{\alpha}:=\{(r, \theta):(1-\alpha) R<r<(1+\alpha) R, 0 \leqslant$ $\theta<2 \pi$, provided $\alpha$ and $\delta$ are chosen sufficiently small. To this end, we introduce the set $S$ via

$$
S:=\left\{\theta \in[0,2 \pi):(r, \theta) \in \partial_{M} A \text { for some } r \in((1-\alpha) R,(1+\alpha) R)\right\} .
$$

Then for any $\theta \notin S$, the entire segment of points $(r, \theta)$ with $r \in((1-\alpha) R,(1+\alpha) R)$ either lies entirely in $A$ or entirely in its complement. Hence half of such a segment lies in $B(0, R) \triangle A$ and from (5.32) we find

$$
\alpha R \mathcal{H}^{1}([0,2 \pi)-S) \leqslant|B(0, R) \triangle A|<\delta .
$$

Consequently,

$$
\mathcal{H}^{1}(S)>2 \pi-\frac{\delta}{\alpha R} .
$$

Inequality (5.34) says that in $\mathcal{A}_{\alpha}, \partial_{M} A$ projects almost fully onto $\partial B(0, R)$.

With (5.34) in hand, it is intuitively clear that $\partial_{M} A$ must have nearly $2 \pi R$ in perimeter when restricted to the annulus $\mathcal{A}_{\alpha}$. This can be established rigorously by, for example, viewing $A$ as a 2current and then viewing $\partial_{M} A$ restricted to the annulus $\mathcal{A}_{\alpha}$ as a 1 -current, say $T$. Inequality (5.34) says that $\partial_{M} A$ intersects most level sets of the function $f(r, \theta):=\theta$ within the thin annulus. Phrased in terms of currents, this means that the mass of the slice of $T$ by $f$, denoted by $M(\langle T, f, \theta\rangle)$, is at least 1 for $\theta \in S$. Then we invoke the inequality $\left[2.8\right.$, noting that $\sup _{(r, \theta) \in \mathcal{A}_{\alpha}}|\nabla f|=1 /(1-\alpha) R$, along with (5.34) to conclude that

$$
\mathcal{H}^{1}\left(\partial_{M} A \cap \mathcal{A}_{\alpha}\right)=M(T) \geqslant 2 \pi R(1-\alpha)-\frac{\delta}{\alpha} .
$$


Next we argue that the perimeter of $A$ will exceed $2 \pi R$ if $\partial_{M} A$ meets a substantial fraction of the circles $\partial B(0, r)$ for $r>(1+\alpha) R$. For example, suppose to the contrary, that for say half of the $r$-values in the interval $(1+\alpha) R<r<1$, one has the condition $\partial_{M} A \cap \partial B(0, r) \neq \emptyset$. Then necessarily

$$
\mathcal{H}^{1}\left(\partial_{M} A \cap\{(r, \theta):(1+\alpha) R<r<1\}\right) \geqslant \frac{1}{2}(1-(1+\alpha) R) .
$$

(This estimate can also be established by slices as was done in obtaining 5.35 , though one would use circular slices with $f$ above replaced by $g(r, \theta)=r$.) Let us now make the choices

$$
\alpha=\left(\frac{1}{8 \pi+2}\right)\left(\frac{1-R}{R}\right) \quad \text { and } \quad \delta=\frac{1}{8}(1-R) \alpha .
$$

There is of course flexibility in selecting $\alpha$ and $\delta$, but with these values, one can sum 5.35 and 5.36 to conclude after a little algebra that

$$
\begin{aligned}
P\left(A, \mathbb{T}^{2}\right) & \geqslant \mathcal{H}^{1}\left(\partial_{M} A \cap \mathcal{A}_{\alpha}\right)+\mathcal{H}^{1}\left(\partial_{M} A \cap\{(r, \theta):(1+\alpha) R<r<1\}\right) \\
& \geqslant 2 \pi R+\frac{1}{8}(1-R)>P\left(B(0, R), \mathbb{T}^{2}\right)
\end{aligned}
$$

We are left to examine the situation where $\partial_{M} A$ fails to intersect at least half of the circles of radius $r \in((1+\alpha) R, 1)$. In this case, there must exist a radius $r_{0}$ with $(1+\alpha) R<r_{0} \leqslant$ $1-\frac{1}{2}[1-(1+\alpha) R]$ such that $\partial_{M} A \cap \partial B\left(0, r_{0}\right)=\emptyset$. Furthermore, one must have the condition $A \cap \partial B\left(0, r_{0}\right)=\emptyset$ as well. Otherwise, $\partial_{M} A$ would have to intersect most rays $\left\{(r, \theta): r_{0}<r<1\right\}$ in order to keep the measure of the symmetric difference $B(0, R) \triangle A$ small and this would in turn drive the perimeter of $A$ above $2 \pi R$ when combined with (5.35).

Examining the conditions $A \cap \partial B\left(0, r_{0}\right)=\partial_{M} A \cap \partial B\left(0, r_{0}\right)=\emptyset$, we can now consider the components of $A$ within and outside of the circle $\partial B\left(0, r_{0}\right)$ separately. If there are no components of $A$ outside this circle, then we are reduced to the standard isoperimetric inequality on the plane and so the only competitor $A$ matching the perimeter of $B(0, R)$ would be a nearby translate of the ball. If, on the other hand, $A$ has components outside of the circle $\partial B\left(0, r_{0}\right)$, then (5.32) forces the measure of $A \cap\left(\mathbb{T}^{2} \backslash B\left(0, r_{0}\right)\right)$ to be small. Again, in this case one knows a better competitor outside of $B\left(0, r_{0}\right)$ would be a ball of measure $\left|A \cap\left(\mathbb{T}^{2} \backslash B\left(0, r_{0}\right)\right)\right|$ so we can lower perimeter by replacing $A$ outside of $B\left(0, r_{0}\right)$ with such a small ball. Likewise, since the components of $A$ are separated by the circle $\partial B\left(0, r_{0}\right)$ one can improve the competitor $A$ inside the ball by replacing it with a ball, say centered at the origin, whose area equals $\left|A \cap B\left(0, r_{0}\right)\right|$. But clearly the single ball $B(0, R)$ has less perimeter than the total perimeter of two balls whose total measure is also $\pi R^{2}$. We have now exhausted all possibilities and the proof is complete.

COROLlaRY 5.6 There exists a value $\varepsilon_{0}>0$ and a sequence $\left\{u_{\varepsilon}\right\}$ of local minimizers of $E_{\varepsilon}$ such that $d_{L^{1}}\left(u_{\varepsilon}, S_{2}\right) \rightarrow 0$ as $\varepsilon \rightarrow 0$, where the set $S_{2}$ is given by 5.33 . Furthermore, for any sequence $\left\{\varepsilon_{j}\right\} \rightarrow 0$, there exists a subsequence $\left\{\varepsilon_{j_{k}}\right\}$ and an element $u$ of $S_{2}$ such that $u_{\varepsilon_{j_{k}}} \rightarrow u$ in $L^{1}$.

Proof. The corollary follows immediately from Propositions 3.2 and 5.5

REMARK 5.7 Besides collections of strips, a case that has been completely exhausted through Proposition 5.1 and Remarks 5.3 and 5.4 the only other critical points of the 2-d (PIP) we have not yet discussed are sets consisting of two or more congruent discs. It is elementary to check that these critical points are never stable, and therefore certainly not local minimizers. For example, one can choose $f$ in the second variation (5.22) to be 1 on one boundary component (circle) and -1 on any 
other to yield a negative second variation. In fact, this type of reasoning goes to show that a stable phase boundary must be connected unless its curvature is everywhere zero in any dimension (see Remark 7.2).

To sum up, through Propositions 5.1, 5.5 and Remarks 5.3, 5.4 and 5.7, we have found all stable critical points of (PIP) in 2-d. They are all found to be $L^{1}$-local minimizers in the sense of (3.16). Except for the case of more multi-component strips, these local minimizers are in fact strict in the sense of Proposition 3.2, thus leading to diffuse analogues.

As we have indicated, the situation in 3-d is far more complicated. The case of 2-d multicomponent strips suggests that in 3-d, stability alone will again not be sufficient to guarantee a strict $L^{1}$-local minimizer in the sense of Proposition 3.2. It is therefore quite likely that we will need some notion of strict stability, e.g. a second variation with a positive lower bound for nontranslational variations.

\section{Small nonlocal perturbations}

Following our discussions in Section 1, we formally introduce the nonlocal analogues of (PCH) and (PIP). Here, we will simply note the relationships between the local and nonlocal problems when the relevant parameters are small.

For $\epsilon>0$ and $m \in(-1,1)$, we define

$$
\mathcal{E}_{\epsilon, \gamma}:= \begin{cases}\int_{\mathbb{T}^{n}}\left(\frac{\epsilon}{2}|\nabla u|^{2}+\frac{1}{\epsilon} \frac{\left(1-u^{2}\right)^{2}}{4}+\frac{\gamma}{2}|\nabla v|^{2}\right) \mathrm{d} x & \text { if } u \in H^{1}\left(\mathbb{T}^{n}\right) \text { and } \int_{\mathbb{T}^{n}} u=m, \\ +\infty & \text { otherwise, }\end{cases}
$$

where $c_{0}$ is a positive constant. Here $v$ is related to $u$ and $m$ via

$$
-\Delta v=u-m \quad \text { on } \mathbb{T}^{n}, \quad \int_{\mathbb{T}^{n}} v(x) \mathrm{d} x=0 .
$$

Note that the third term in 6.37 represents a compact perturbation with respect to the basic $L^{2}$ (or $L^{1}$ ) topology. Hence it easily follows (cf. [43]) from the definition of $\Gamma$-convergence (cf. [7]) that the $\Gamma$-limit is

$$
\mathcal{E}_{0, \gamma}:= \begin{cases}c_{0} \int_{\mathbb{T}^{n}}\left(|\nabla u|+\frac{\gamma}{2}|\nabla v|^{2}\right) \mathrm{d} x & \text { if } u \in B V\left(\mathbb{T}^{n}\right),|u|=1 \text { a.e., } \int_{\mathbb{T}^{n}} u=m, \\ +\infty & \text { otherwise. }\end{cases}
$$

The nonlocal Cahn-Hilliard and isoperimetric problems are defined as follows:

$(\mathrm{NLCH}) \quad$ Minimize $\mathcal{E}_{\epsilon, \gamma}(u)$ over all $u \in L^{1}\left(\mathbb{T}^{n}\right)$,

(NLIP) Minimize $\mathcal{E}_{0, \gamma}(u)$ over all $u \in L^{1}\left(\mathbb{T}^{n}\right)$.

The coefficient $c_{0}$ plays no role and in what follows we set $c_{0}=1$.

Following the discussion of the introduction (see the references therein), 6.37 and 6.38) can be viewed as models for periodic phase separation induced by long and short-range energetic competitions. As with (PCH) and (PIP), we have chosen to adopt periodic boundary conditions, and 
hence any minimizer can still be regarded as periodic with period one. However, in this instance, the choice is simply for convenience. As regards periodicity, what is significant here is that for $\gamma$ sufficiently large, a smaller scale is enforced as a weak constraint via interactions between the perimeter and the nonlocal terms. One conjectures (see the discussion in the introduction) that minimizers are nearly periodic, and it is this inherent mesoscopic peridocity which makes this functional of interest. Here we are interested in the regime of small $\gamma$, and the connection with $(\mathrm{PCH})$ and (PIP) - this is naturally facilitated by the universal adoption of periodic boundary conditions.

For small $\gamma$, one would expect the effect of the nonlocal term to be minimal. In fact, this has been confirmed by Ren and Wei (see for example [45-47]), where in 2-d for $\gamma$ small, it has been shown that some simple solutions to (PCH) and (PIP) are still stable (for example, a circular disc). In the same spirit, we note that one can easily obtain the following asymptotic result.

Proposition 6.1 Let $n=2,3$ and $-1<m<1$. Let $\eta>0$. There exist $\epsilon_{0}>0$ and $\gamma_{0}>0$ such that for all $\epsilon<\epsilon_{0}$ and $\gamma<\gamma_{0}$, if $u_{\epsilon, \gamma}$ is a minimizer of (NLCH) then

$$
\inf _{y \in \mathbb{T}^{n}}\left\|u_{\epsilon, \gamma}(\cdot-y)-u^{*}\right\|_{L^{1}\left(\mathbb{T}^{2}\right)}<\eta,
$$

where $u^{*}$ is a minimizer of (PIP).

An analogous result to Proposition 6.1 also holds for $L^{1}$-local minimizers.

Proof. Suppose 6.39 is false. Then there exist $\eta_{0}>0$ and sequences $\left\{\epsilon_{j}\right\},\left\{\gamma_{k}\right\}$ tending to 0 such that

$$
\inf _{y \in \mathbb{T}^{n}}\left\|u_{\epsilon_{j}, \gamma_{k}}(\cdot-y)-u^{*}\right\|_{L^{1}\left(\mathbb{T}^{n}\right)} \geqslant \eta_{0}, \quad \forall j, k .
$$

It is trivial to verify that

$$
\mathcal{E}_{0, \gamma} \Gamma \text {-converges to } \mathcal{E}_{0,0} \quad \text { as } \gamma \rightarrow 0 \text {. }
$$

Note that $\mathcal{E}_{0,0}$ is simply $E_{0}$ as defined in 3.10 . Hence, for every $\eta>0$, there exists $\gamma(\eta)$ such that for all $\gamma<\gamma(\eta)$ and $v_{\gamma}$ minimizing $\mathcal{E}_{0, \gamma}$, one has

$$
\inf _{y \in \mathbb{T}^{n}}\left\|v_{\gamma}(\cdot-y)-u^{*}\right\|_{L^{1}\left(\mathbb{T}^{n}\right)} \leqslant \eta
$$

Now fix $k$ such that $\gamma_{k}<\gamma\left(\eta_{0} / 2\right)$. Since for any $\gamma$,

$$
\mathcal{E}_{\epsilon, \gamma} \Gamma \text {-converges to } \mathcal{E}_{0, \gamma} \quad \text { as } \epsilon \rightarrow 0,
$$

there exist $j$ and $v_{k}$ minimizing $\mathcal{E}_{0, \gamma_{k}}$ such that

$$
\left\|u_{\epsilon_{j}, \gamma_{j}}-v_{k}\right\|_{L^{1}\left(\mathbb{T}^{n}\right)}<\eta_{0} / 2 .
$$

With these choices of $k$ and $j, 6.41$ and 6.42 contradict 6.40.

Naturally, the interesting regimes will be for $\gamma$ large and it will be here that a mesoscopic scale for the periodicity (alluded to above and in the introduction) is weakly enforced by energetic competitions. This regime is the focus of [2] and [12]. 


\section{Remarks}

\subsection{Open problems}

Returning to (PCH) and (PIP), we summarize some important remaining questions mentioned in this article.

(i) Conjecture 4.5 remains a basic assertion regarding the classification of global minimizers of (PIP) according to the volume constraint for the cubic flat 3-torus. For other flat 3-tori, one would like to have similar characterizations.

(ii) If one could establish a general principle stating that sets possessing (strictly) stable CMC boundaries must necessarily locally minimize (PIP) in the $L^{1}$ sense of Proposition 3.2 then it would open the door to a rich new collection of local minimizers of the 3-d (PCH). Along with this, we would like a better understanding of which stable surfaces are isolated in the sense of Proposition 3.2. Perhaps a natural approach to this problem would be via a calibration-like method whereby one foliates a neighborhood of a stable surface with CMC surfaces. Needless to say, one would benefit from a more exhaustive study of triply periodic stable surfaces, especially within the context of notions of strict stability.

\subsection{Connectivity of the phase boundary}

Returning to the model problem of microphase separation of diblock copolymers, many structures have been observed which have discontinuous phase boundaries. An interesting case in point is the double gyroid (cf. [6, 19, 28]). A similar structure with a diffuse interface has also been observed in numerical simulations for minimizers of $(\mathrm{NLCH})$ (cf. [55]). It is worth noting that these structures are not stable solutions of (PIP). This follows immediately from consideration of the second variation. Indeed, as long as $\left\|B_{\Sigma}\right\| \not \equiv 0$, any multi-component surface $\Sigma=\Sigma_{1} \cup \Sigma_{2}$ would be unstable, as can be seen by substituting

$$
f= \begin{cases}1 & \text { on } \Sigma_{1}, \\ -\mathcal{H}^{n-1}\left(\Sigma_{1}\right) / \mathcal{H}^{n-1}\left(\Sigma_{2}\right) & \text { on } \Sigma_{2},\end{cases}
$$

into 5.22). One might also ask whether local minimizers of $(\mathrm{PCH})$ can have multi-component transition layers where say $u_{\varepsilon} \approx 0$. For the case of Neumann boundary conditions in convex domains, the answer was shown to be no in [54] and we suspect the same is true in this periodic setting but we have not checked the details.

\subsection{Varifolds and convergence of the phase boundaries}

We are grateful to Y. Tonegawa for commenting that in the spirit of [32, 57, 58], one should be able to use the notion of an associated varifold (cf. [52]) to prove a stronger result on the convergence of phase boundaries. Loosely speaking, a varifold associated to a minimizing sequence $u_{\epsilon}$ of (PCH) is a weighted average of the level sets of $u_{\epsilon}$, concentrating around the transition layer. There are many advantages to this type of convergence, for example it yields information on the distribution of energy as well as geometric and analytic information about the transition layer (see [32, 57, 58] for more details). 


\section{Acknowledgments}

The authors are grateful to A. Ros for a useful correspondence on the subject matter.

\section{REFERENCES}

1. Aksimentiev, A., Fialkowski, M., \& Holyst, R. Morphology of surfaces in mesoscopic polymers, surfactants, electrons, or reaction-diffusion systems: methods, simulations, and measurements. Adv. Chem. Phys. 121 (2002), 141-239.

2. Alberti, G., Choksi, R., \& Oтto, F. Uniform energy distribution for minimizers of a nonlocal functional. In preparation.

3. Alberti, G., \& Müller, S. A new approach to variational problems with multiple scales. Comm. Pure Appl. Math. 54 (2001), 761-825. Zbl 1021.49012 MR 1823420

4. Anderson, D., Davis, H., Scriven, L., \& Nitsche, J. C. Periodic surfaces of prescribed mean curvature. Adv. Chem. Phys. 77 (1990), 337.

5. Andersson, S., Hyde, S. T., Larsson, K., \& Lidin, S. Minimal surfaces and structures: from inorganic and metal crystals to cell membranes and biopolymers. Chem. Rev. 88 (1988), 221-242.

6. Bates, F. S., \& Fredrickson, G. H. Block copolymers-designer soft materials. Physics Today 52 (1999), no. 2, 32-38.

7. Braides, A. $\Gamma$-convergence for Beginners. Oxford Lecture Ser. Math. Appl. 22, Oxford Univ. Press (2002). Zbl pre01865939 MR 1968440

8. BRAKKe, K. The surface evolver. Experiment. Math. 1 (1992), 141-165. Software available at: http://www.susqu.edu/facstaff/b/brakke/evolver/evolver.html.

9. Cahn, J. W., \& Hilliard, J. E. Free energy of a nonuniform system I. Interfacial Free Energy. J. Chem. Phys. 28 (1958), 258-267.

10. CHOKsI, R. Scaling laws in microphase separation of diblock copolymers. J. Nonlinear Sci. 11 (2001), 223-236. Zbl 1023.82015 MR 1852942

11. Choksi, R., \& REN, X. On a derivation of a density functional theory for microphase separation of diblock copolymers. J. Statist. Phys. 113 (2003), 151-176. Zbl 1034.82037 MR 2012976

12. Choksi, R., \& Sternberg, P. On the first and second variations of a nonlocal isoperimetric problem. J. Reine Angew. Math., to appear.

13. Cochran, E. W., Garcia-Cevera, C. J., \& Fredrickson, G. H. Stability of the gyroid phase in diblock copolymers at strong segregation. Preprint (2006).

14. Davidovitch, B., Ertas, D., \& Halsey, T. C. Ripening of porous media. Phys. Rev. E 70 (2004), 031609.

15. Davidovitch, B., Ertas, D., \& Halsey, T. C. Reaction-limited sintering in nearly saturated environments. Philos. Mag. 84 (2004), 1937.

16. Federer, H. Geometric Measure Theory. Springer, New York (1969). Zbl 0874.49001 MR 0257325

17. FIFE, P. Models for phase separation and their mathematics. Electron. J. Differential Equations 2000, no. 48, 1-26. Zbl 0957.35062 MR 1772733

18. Fife, P., Kielhöfer, H., Maier-PaApe, S., \& Wanner, T. Perturbation of doubly periodic solution branches with applications to the Cahn-Hilliard equation. Phys. D 100 (1997), 257-278. Zbl 0890.35043 MR 1429160

19. Förster S., Khandpur, K., Zhao, J., Bates, F., Hamley, I., Ryan, J., \& Bras, W. Complex phase behavior of polyisoprene-polystyrene diblock copolymers near the order-disorder transition. Macromolecules 27 (1994), 6922-6935.

20. GiUsti, E. Minimal Surfaces and Functions of Bounded Variation. Birkhäuser (1985). 
21. Gonzalez, E., Massari, U., \& TAMAnini, I. On the regularity of boundaries of sets minimizing perimeter with a volume constraint. Indiana Univ. Math. J. 32 (1983), 25-37. Zbl 0486.49024 MR 0684753

22. Grosse-Brauckmann, K. Stable constant mean curvature surfaces minimize area. Pacific J. Math. 175 (1997), 527-534. Zbl 0878.49026 MR 1432843

23. Grosse-Brauckmann, K. Gyroids of constant mean curvature. Experiment. Math. 6 (1997), 33-50. Zbl 0898.53010 MR 1464580

24. Grosse-Brauckmann, K. On gyroid interfaces. J. Colloid Interface Sci. 187 (1997), 418-428.

25. Grosse-Brauckmann, K., \& Wohlgemuth, M. The gyroid is embedded and has constant mean curvature. Calc. Var. Partial Differential Equations 4 (1996), 499-523. Zbl 0930.53009 MR 1415998

26. Gurtin, M. On a theory of phase transitions with interfacial energy. Arch. Ration. Mech. Anal. 87 (1985), no. 3, 187-212. MR 0768066

27. Hadwiger, H. Gitterperiodische Punktmengen und Isoperimetrie. Monatsh. Math. 76 (1972), 410-418. Zbl 0248.52012 MR 0324550

28. HAJduk, D. A. ET AL. The gyroid: a new equilibrium morphology in weakly segregated diblock copolymers. Macromolecules 27 (1994), 4063-4075.

29. Hauswirth, L., Perez, J., Romon, P., \& Ros, A. The periodic isoperimetric problem. Trans. Amer. Math. Soc. 356 (2004), 2025-2047. Zbl 1046.52002 MR 2031051

30. Hoffman, D. A new turn for Archimedes. Nature 384 (1996), 28-29.

31. Howards, H., Hutchings, M., \& Morgan, F. The isoperimetric problem on surfaces. Amer. Math. Monthly 106 (1999), 430-439. Zbl 1003.52011 MR 1699261

32. Hutchinson, J., \& Tonegawa, Y. Convergence of phase interfaces in the van der WaalsCahn-Hilliard theory. Calc. Var. Partial Differential Equations 10 (2000), 49-84. Zbl 1070.49026 MR 1803974

33. KARCHER, H. The triply periodic minimal surfaces of Alan Schoen and their constant mean curvature companions. Manuscripta Math. 64 (1989), 291-357. Zbl $0687.53010 \mid$ MR 1003093

34. Kenmotsu, K. Surfaces with Constant Mean Curvature. Transl. Math. Monogr. 221, Amer. Math. Soc. (2003). Zbl 1042.53001 MR 2013507

35. Kohn, R. V., \& Sternberg, P. Local minimisers and singular perturbations. Proc. Roy. Soc. Edinburgh Sect. A 111 (1989), 69-94. Zbl 0676.49011 MR 0985990

36. ModicA, L. The gradient theory of phase transitions and the minimal interface criterion. Arch. Ration. Mech. Anal. 98 (1987), 123-142. Zbl 0616.76004 MR 0866718

37. Modica, L., \& Mortola, S. Un essempio di $\Gamma^{-}$-convergenza. Boll. Un. Mat. Ital. (5) 14 (1977), 285-299. Zbl 0356.49008 MR 0445362

38. MÜLLER, S. Singular perturbations as a selection criterion for periodic minimizing sequences. Calc. Var. 1 (1993), 169-204. Zbl 0821.49015 MR 1261722

39. Muratov, C. B. Theory of domain patterns in systems with long-range interactions of Coulomb type. Phys. Rev. E 66 (2002), 066108. MR 1953930

40. NishiurA, Y., \& OHNishi, I. Some mathematical aspects of the micro-phase separation in diblock copolymers. Phys. D 84 (1995), 31-39. MR 1334695

41. Nitsche, J. C. C. Lectures on Minimal Surfaces I. Cambridge Univ. Press (1989). Zbl 0688.53001 MR 1015936

42. Ohta, T., \& KaWASAKI, K. Equilibrium morphology of block copolymer melts. Macromolecules 19 (1986), 2621-2632.

43. REN, X., \& WEI, J. On the multiplicity of solutions of two nonlocal variational problems. SIAM J. Math. Anal. 31 (2000), 909-924. Zbl 0973.49007 MR 1752422 
44. REN, X., \& WEI, J. On energy minimizers of the diblock copolymer problem. Interfaces Free Bound. 5 (2003), 193-238. Zbl 1031.49035 MR 1980472

45. REN, X., \& WEI, J. Wriggled lamellar solutions and their stability in the diblock copolymer problem. SIAM J. Math. Anal. 37 (2005), 455-489. MR 2176111

46. REN, X., \& WeI, J. Stability of spot and ring solutions of the diblock copolymer equation. J. Math. Phys. 45 (2004), 4106-4133. Zbl 1064.82040 MR 2098120

47. REN, X., \& WEI, J. Concentrically layered energy equilibria of the diblock copolymer problem. Europ. J. Appl. Math. 13 (2002), 479-496. Zbl 1010.82041 MR 1939157

48. Ritore M., \& Ros, A. Stable constant mean curvature tori and the isoperimetric problem in three space forms. Comment. Math. Helvet. 67 (1992), 293-305. Zbl 0760.53037 MR 1161286

49. Ros, A. The isoperimetric problem. Proceedings from the Clay Mathematics Institute Summer School, MSRI, Berkeley, CA. Available at www.ugr.es/ aros.

50. Ross, M. Schwarz' P and D surfaces are stable. Differential Geom. Appl. 2 (1992), 179-195. Zbl 0747.53010 MR 1245555

51. The Scientific Graphics Project. http://www.msri.org/publications/sgp/SGP/indexc.html

52. Simon, L. Lectures on Geometric Measure Theory. Proc. Centre Math. Anal. Austral. Nat. Univ. 3 (1983). Zbl 0546.49019 MR 0756417

53. Sternberg, P. The effect of a singular perturbation on nonconvex variational problems. Arch. Ration. Mech. Anal. 101 (1988), 209-260. Zbl 0647.49021 MR 0930124

54. Sternberg, P., \& Zumbrun, K. Connectivity of phase boundaries in strictly convex domains. Arch. Ration. Mech. Anal. 141 (1998), 375-400. Zbl 0911.49025 MR 1620498

55. Teramoto, T., \& Nishiura, Y. Double gyroid morphology in a gradient system with nonlocal effects. J. Phys. Soc. Japan 71 (2002), 1611-1614.

56. Thomas, E., Anderson, D. M., Henkee, C. S., \& Hoffman, D. Periodic area-minimizing surfaces in block copolymers. Nature 334 (1988), 598-601.

57. Tonegawa, Y. Phase field model with a variable chemical potential. Proc. Roy. Soc. Edinburgh Sect. A 132 (2002), 993-1019. Zbl 1013.35070 MR 1926927

58. Tonegawa, Y. A diffused interface whose chemical potential lies in a Sobolev space. Ann. Scuola Norm. Sup. Pisa 4 (2005), 487-510. MR 2185866

59. White, B. A strong minimax property of nondegenerate submanifolds. J. Reine Angew. Math. 457 (1994), 203-218. Zbl 0808.49037 MR 1305283

60. YIP, N. K. Structure of stable solutions of a one-dimensional variational problem. Preprint (2005). 